This is the author's copy of the publication as archived with the DLR's electronic library at http://elib.dlr.de . Please consult the original publication for citation, see e.g. https://journals.sagepub.com/doi/10.1177/0954409721993626

\title{
Identification of a dynamic friction model for railway disc brakes
}

\section{Ehret}

Braking forces occurring during emergency brake applications of passenger trains are generated by disc brake units. The acting friction forces depend on the frictional properties between disc and brake pad and are influenced by relative velocity, temperatures and normal pressure of the contacting surfaces. In this work a mathematical model is developed which aims to link these influencing variables to the instantaneous acting friction coefficient in order to include the characteristic behavior of friction forces in the calculation of longitudinal dynamics of railway vehicles. The model is identified by the use of data recorded on a full-scale dynamometer test rig and verified regarding the estimation of the brake distance.

\section{Copyright Notice}

Copyright (C 2021 by German Aerospace Center (DLR). This is the accepted version for publication at Proceedings of the Institution of Mechanical Engineers, Part F: Journal of Rail and Rapid Transit.

Ehret M. Identification of a dynamic friction model for railway disc brakes. Proceedings of the Institution of Mechanical Engineers, Part F: Journal of Rail and Rapid Transit. February 2021. doi: $10.1177 / 0954409721993626$ 


\title{
Identification of a dynamic friction model for railway disc brakes
}

\author{
Marc Ehret \\ German Aerospace Center, Institute of System Dynamics and Control \\ Münchner Straße 20,82234 Weßling, Germany (E-mail: marc.ehret@dlr.de)
}

\begin{abstract}
Braking forces occurring during emergency brake applications of passenger trains are generated by disc brake units. The acting friction forces depend on the frictional properties between disc and brake pad and are influenced by relative velocity, temperatures and normal pressure of the contacting surfaces. In this work a mathematical model is developed which aims to link these influencing variables to the instantaneous acting friction coefficient in order to include the characteristic behavior of friction forces in the calculation of longitudinal dynamics of railway vehicles. The model is identified by the use of data recorded on a full-scale dynamometer test rig and verified regarding the estimation of the brake distance.
\end{abstract}

KEYWORDS: dynamic model, friction coefficient, railway disc brakes, dynamometer, numerical optimization 


\section{Introduction}

The brake distance is a key requirement which a brake system of a railway vehicle has to meet, especially in emergency cases. Its early prediction is an important task during the development of railway brake systems and is conducted by applying calculations from the very beginning of the design based on standards, such as DIN EN 14531-1/2 [1] [2]. In these calculations frictional forces of disc brakes are usually taken into account by using a constant mean value as soon as the brake cylinders are fully actuated. However, measurements recorded during emergency brake applications of railway vehicles reveal that the deceleration of the train may vary strongly after the maximum pressure in the brake cylinders is reached. For a passenger train equipped with pneumatically actuated wheel-disc brakes this behavior is shown in Figure 1. The measured deceleration curves of different emergency brake applications with varying initial velocities are characterized by a mostly increasing progression. Furthermore, the curves indicate that the initial velocity has a major influence on the rate of growth during the brake phase. An increasing initial velocity seems to yield a decreasing maximum deceleration. In case of $v_{0}=100 \mathrm{~km} / \mathrm{h}$ the initial deceleration changes by more than $30 \%$, whereas a change of less than $20 \%$ is observed for $v_{0}=160 \mathrm{~km} / \mathrm{h}$.

As one can see from the first seconds in Figure 1 the deceleration caused by resistance forces is small in comparison to the total deceleration when the disc brakes are acting. In addition, the measurements are recorded on a flat and straight track and the driving resistance forces are decreasing with declining velocity. It follows that the brake system is mainly responsible for the varying deceleration. Furthermore, the measurements were conducted on regular track conditions and therefore the acting tangential contact forces between wheel and rail during braking were smaller than the limits of adhesion. Thus, no macroscopic skidding of the wheels on the track was present during braking. The change of deceleration is therefore caused by the brake torques of the disc brakes. However, the brake cylinder pressure and accordingly the normal forces acting on the brake pads are independent of the initial velocity and remain constant as soon as the maximum value is reached, as shown in Figure 2. In conclusion, the root cause of the varying brake torques must be linked to the frictional forces transmitted at the surface of the disc brakes. 


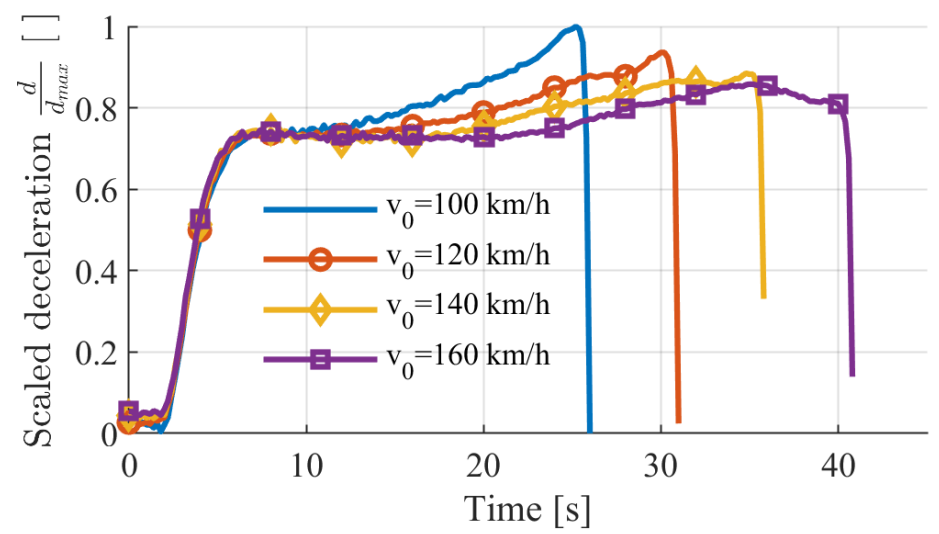

Figure 1: Scaled representation of measured deceleration during emergency brake applications of a passenger train with varying initial velocities

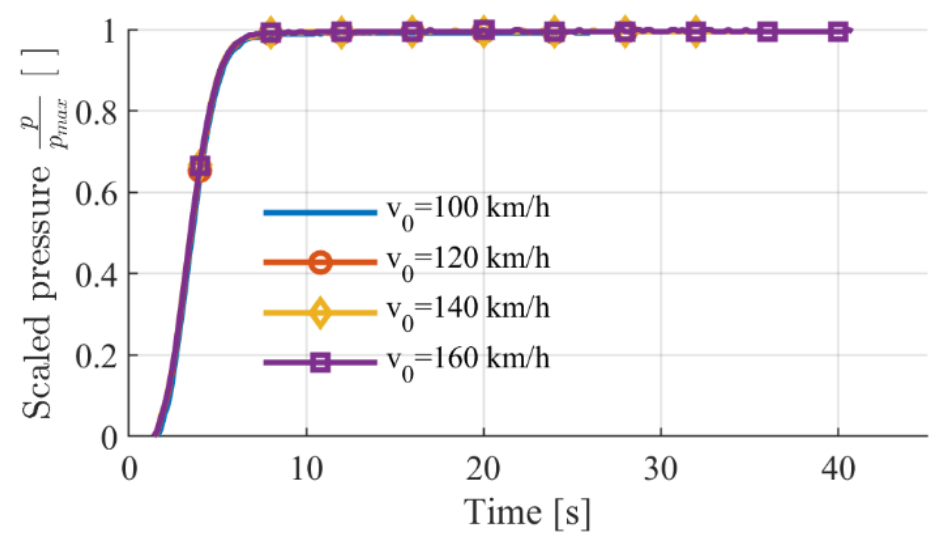

Figure 2: Scaled representation of measured brake cylinder pressure during emergency brake applications of a passenger train with varying initial velocities

During braking the frictional boundary layer between disc and pads is exposed to high thermo-mechanical loads and relative movement. In this case more than $20 \mathrm{kN}$ are pressing the single pads against the disc and the maximum frictional velocity at the beginning of the brake phase ranges from $65 \mathrm{~km} / \mathrm{h}$ to $105 \mathrm{~km} / \mathrm{h}$. Depending on the transformed amount of energy, these external loads and operating conditions induce a change of frictional properties between pad and disc that is responsible for the varying brake torques. The instantaneous deceleration and the resulting brake distance are therefore strongly correlated to the acting friction coefficient. Its early prediction becomes a crucial task for the design of the entire brake system. 
Different empirical approaches have been proposed in [3], [4] and [5] to estimate the instantaneous friction coefficient of railway vehicles based on numerical simulation. With the exception of [5], however, these approaches have their origin in the analysis of railway tread brakes and not disc brakes. Furthermore, all approaches are based on the imitation of the friction forces and lack a deeper analysis of the actual friction phenomena of disc brakes. This work aims to fill this gap by identifying a friction model that explains the characteristic behavior of railway disc brakes based on physical friction phenomena.

The goal of this work is to derive a mathematical model for the instantaneous friction coefficient based on measurements recorded during a standard testing procedure of a wheel-mounted disc brake on a full-scale dynamometer test rig. The chosen approach interconnects the rate of change of the friction coefficient between brake pad and disc with growth and destruction of contact patches in the frictional boundary layer. The idea of the presented concept is based on the work of Ostermeyer [6] [7] in the context of automotive applications and requires the identification of terms primarily influencing the frictional properties of railway disc brakes. A nonlinear, first order differential equation is derived which correlates the change of friction coefficient to mechanical and thermal loads.

In Chapter 2 an overview of different mathematical models for the numerical simulation of friction phenomena is given and the chosen method is presented. In Chapter 3 the measurement set-up and data are presented. Subsequently, the data is used in Chapter 4 to identify the model and its parameters based on emergency brake applications with varying initial velocities and external loads. The model parameters are tuned by the use of numerical optimization. In order to verify the results, the calculated brake distances are compared to the measured ones in Chapter 5. Finally, a conclusion and an outlook for future work are given. 


\section{Friction models}

\subsection{General approaches for numerical simulation}

Friction phenomena are the result of many different mechanisms that occur between contacting bodies depending on surface topography, contact geometry and materials, displacement, relative velocity, wear, normal contact forces, temperature, adhesion and lubrication [8]. Consequently, there is a huge variety of models that aim to describe tangential friction forces for different applications, scales and domains. In [9] and [10] an overview of the variety of models applied in numerical simulation is given. Starting from classical models, such as Coulomb Friction that connects the tangential friction force $F_{T}$ with the normal force $F_{N}$ by the friction coefficient $\mu$

$$
F_{T}=\mu \cdot F_{N},
$$

more sophisticated models are presented, e.g. Armstrong's Model. These models differentiate between sliding and sticking and consider the dependency of friction on the relative velocity. In contrast to quasistatic models, which are mostly algebraic equations, dynamic models consider transition regions between different regimes to be continuous by introducing differential equations for the friction force. Beside the enhanced eligibility for the numerical solution, dynamic models are able to capture the transfer behavior between friction forces and variable external loads.

\subsection{Modelling approaches for disc brakes}

Friction phenomena occurring in disc brakes of ground vehicles are primarily characterized by the large amount of energy transformed in the contact region during braking. Therefore, disc and brake pad are exposed to large ranges of frictional velocity and external loads, such as frictional and normal forces as well as temperatures. Beside the resulting varying friction forces, a dependency of frictional properties on the load history might occur, as analyzed in [11]. Furthermore, vehicle disc brakes operate under environmental conditions including moisture, ice, impurities and vibrations, which influence the frictional properties. Additionally, brake squeal and creaking induced by stick-slip effects and self-induced vibrations are common problems closely linked to the frictional behavior of disc brakes. The briefly described complexity of the brake process yields a broad spectrum of friction models in order to analyze various problems of disc brakes, as discussed in [12]. The numerical analysis of longitudinal dynamics of vehicles 
during braking is in general performed by applying the Coulomb Friction Model in equation (1) for disc brake forces in conjunction with a variable or constant friction coefficient. This is an effective method to include complex friction processes depending on diverse external loads in calculation models. Table 1 shows different static and dynamic approaches to model the friction coefficient applied for friction brakes of railway and automotive vehicles.

Table 1: Models for the friction coefficient of disc and tread brakes of railway vehicles (Rail.) and disc brakes of automotive vehicles (Auto.). The meaning of the parameters is given in the references.

\begin{tabular}{|c|c|c|}
\hline Model & Formula for friction coefficient $\mu$ & Appl. \\
\hline Constant [1] & $\mu=\mu_{\text {mean }}$ & Rail. \\
\hline Karwatzki [4] & $\mu=k_{1} \cdot\left(\frac{F_{N}+k_{2}}{F_{N}+k_{3}}\right) \cdot\left(\frac{v+k_{4}}{v+k_{5}}\right)$ & Rail. \\
\hline Gralla [4] & $\mu=A \cdot P \cdot V \cdot W$ & Rail. \\
\hline Cantone [3] & $\mu=a_{0}+a_{1} \cdot v+a_{2} \cdot v^{2}+a_{3} \cdot v^{3} \ldots$ & Rail. \\
\hline Lee [5] & $\mu=\mu_{0} \cdot\left(n_{v} e^{\left.-m_{v} \cdot v+1\right) \cdot\left(n_{T} e^{-m_{T} \cdot T_{d}}+1\right)}\right.$ & Rail. \\
\hline Rhee [13] & $\mu=k \cdot F_{N}{ }^{a} \cdot v^{b}$ & Auto. \\
\hline Loh [14] & $\mu=a+b \cdot e^{-\frac{t}{c}}$ & Auto. \\
\hline Velimir [15] & $\operatorname{Input~} \rightarrow$ Neural Network $\rightarrow \mu$ & Auto. \\
\hline Ostermeyer [7] & $\dot{\mu}=f_{G}\left(\mu, s_{1}, s_{2}, \ldots\right)-f_{D}\left(\mu, s_{1}, s_{2}, \ldots\right)$ & Auto. \\
\hline
\end{tabular}

In comparison to most of the other approaches the usage of a constant friction coefficient representing the mean value of the coefficient $\mu_{\text {mean }}$ over time, velocity or distance allows for a solution of longitudinal dynamics without numerical integration. However, it is not possible to retrace the time dependent dynamics of the brake forces.

In order to describe brake forces produced by tread brakes of freight trains Karwatzki and Gralla developed empirical formulas for the friction coefficient taking into account normal force $F_{N}$, velocity $v$ and the configuration of train and brake pads [4]. In contrast to the model of Karwatzki, which is a single formula with five parameters $k_{i}$, the model of Gralla consists of four terms $A, P, V$ and $W$, depending on initial and instantaneous velocity, friction work and brake type. Another approach originating from tread brakes is the use of polynomial regression depending on the velocity suggested by Cantone in [3]. In this work two methods are presented in which the parameters $a_{i}$ are fitted to datasets with varying normal forces and 
initial velocity. Although, the frictional behavior in tread brakes varies stronger than in disc brakes during braking [16], the models of Gralla, Karwatzki and Cantone are also applied for the friction coefficient of disc brakes.

Lee proposes in [5] an exponential equation for the friction coefficient that depends on the vehicle speed and, in contrast to the other mentioned approaches in railway applications, on the disc temperature $T_{d}$. As shown in [13], the correlation of disc temperature and friction coefficient in automotive disc brakes has already been proposed by Rhee in 1974. Here, the exponents $a$ und $b$ are a set of parameters depending on the temperature.

Based on the observed dynamics of the friction coefficient in automotive brakes Loh et al. developed a model for the friction coefficient based on a differential equation to describe a time dependent growth process [14]. For each load case, characterized by normal force, initial velocity and material of the brake lining, the parameters of the model $a, b$ and $c$ are fitted individually.

In [15] Velimir analyzes the use of artificial neural networks for the estimation of the friction coefficient of disc brakes. In this approach, a network of artificial neurons is trained to reproduce the friction coefficient depending on input data, such as external loads, environmental conditions and material. The results, however, strongly dependent on the data basis and the network design.

Based on the detailed analysis of the processes taking place in the frictional boundary layer between disc and pad Ostermeyer proposes a model for the derivative of friction coefficient with respect to time $\dot{\mu}$ [7]. Due to a rough topography of the contacting surfaces, forces between pad and disc are primarily transmitted by certain zones of the pad surface [6] [7]. These so-called patches are the actual contact areas between pad and disc. The bigger the contact area, the higher the capability of transmitting frictional forces. It is therefore proposed that the friction coefficient can be linked to the total patch area [6]. The basic idea of the model approach implies that the dynamic of the friction coefficient $\dot{\mu}$ is determined by processes affecting both growth $f_{G}$ and destruction $f_{D}$ of patch areas. As discussed in [7], diverse processes regulate the evolution of patches, which are in turn dependent on influencing factors $s_{i}$, such as the amount of abrasion, temperature of contacting materials or friction power. These are dynamic quantities themselves and might 
be coupled to each other and external loads. Consequently, friction is considered as control loop which can be described by a system of differential equations [7].

\subsection{Model choice}

In comparison to the other discussed models, Ostermeyer's approach is not focusing on the imitation of the measured behavior, but tries to retrace the actual root causes of friction and its dynamics. Consequently, it is assumed that a model based on this approach captures the frictional behavior for multiple load cases without using individual parameters for each load case, as proposed by Gralla, Cantone, Loh and Rhee. In comparison to neural networks and polynomial regression, which are non-physical methods, the application of this model approach additionally supports an enhanced understanding of frictional processes taking place in railway disc brakes. Investigating and retracing these processes seems to be a promising method to provide individual models for commonly applied types of brake pads, discs and materials. Finally, the use of differential equations might enable to consider load history effects, for instance by assigning the initial value of the friction coefficient depending on previous loads. For the above mentioned reasons, a model based on the approach of Ostermeyer shall be developed and identified in this work and verified regarding its suitability for railway disc brakes.

\section{Data basis}

A model based on the chosen approach is identified using measurements from a full-scale dynamometer test rig. The test rig is placed at KNORR-BREMSE SfS GmbH and applied for the evaluation of disc brakes and pads in conjunction with brake caliper units during the design of brake systems according to the requirements of standards, e.g. given in [17], and customers.

\subsection{Measurement set-up}

A simplified sketch of the test rig and the acting forces is drawn in Figure 3. The disc brake or wheel-disc brake is mounted on an axle and connected to the inertia dynamometer in order to simulate an adjustable rotating inertia $m_{e f f}$. The brake torque $T_{B}$ is generated by a single caliper unit and is actuated by the brake cylinder pressure $p_{C}$. 


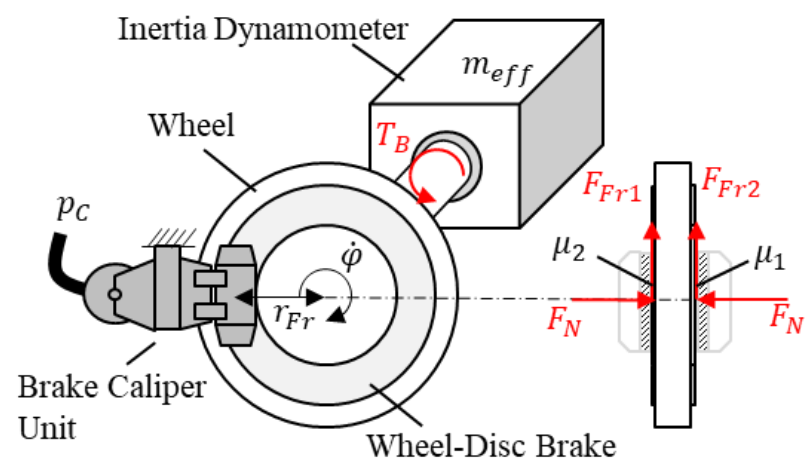

Figure 3: Simplified sketch of dynamometer test rig applied for wheel-disc brake

The force acting on the piston of the brake cylinder is transmitted via the caliper to the normal force $F_{N}$. This force presses the brake pads against each side of the disc. Consequently, the total friction force $F_{F r}$ results from two friction contacts, and thus two coefficients $\mu_{1 / 2}$, respectively.

$$
F_{F r}=F_{F r 1}+F_{F r 2}=F_{N} \cdot\left(\mu_{1}+\mu_{2}\right)
$$

Assuming the friction forces $F_{F r 1 / 2}$ act on the same distance $r_{F r}$ in relation to the center of the axle, the brake torque is given by:

$$
T_{B}=r_{F r} \cdot F_{F r}=r_{F r} \cdot F_{N} \cdot\left(\mu_{1}+\mu_{2}\right)
$$

The estimation of the instantaneous acting friction coefficient using this set-up is based on the measurement of the brake torque and the cylinder pressure, respectively the normal forces:

$$
\mu_{1}+\mu_{2}=\frac{T_{B}}{r_{F r} \cdot F_{N}}
$$

With this set-up it is not possible to measure the single friction forces $F_{F r 1 / 2}$ and therefore to distinguish between $\mu_{1}$ and $\mu_{2}$. Assuming they are equal

$$
\mu_{1}=\mu_{2}=\mu \text {, }
$$

and introducing the total clamping force $F_{C}$, it follows:

$$
\mu=\frac{T_{B}}{r_{F r} \cdot 2 \cdot F_{N}}=\frac{T_{B}}{r_{F r} \cdot F_{C}}
$$

Beside the estimation of the friction coefficient the rotational speed $\dot{\varphi}$ is measured, which is related to the vehicle speed $v$ by the wheel radius $r_{W}$, respectively the friction velocity $v_{F r}$ by the friction radius $r_{F r}$ : 


$$
\dot{\varphi}=\frac{v}{r_{W}}=\frac{v_{F r}}{r_{F r}}
$$

Furthermore, the temperature of the disc is measured at three different radii, located in the middle radius and close to the inner and outer radius. All measurement positions are located close to the surface on both of the disc sides, yielding six measurement positions. These measurements represent the temperature evolution in the disc near the actual friction contact.

\subsection{Measurement data}

The measurement data provided for this work is part of a standard UIC test program, specified in [17]. According to this procedure, emergency brake applications with varying loads, brake modes, effective masses, initial velocities and disc temperatures as well as environmental conditions are performed in a predefined order. The selected measurements simulate a wheel-mounted disc brake, made of cast steel, that operates under dry conditions without an additional electrodynamic brake. The brake pad is a so-called UIC200 dovetail according to [17], which is a standard type applied for railway disc brakes. It is made of sintered copper and iron powder. The basic pad properties are given in Table 2.

Table 2: Basic properties of investigated sinter brake pad

\begin{tabular}{|l|c|l|c|}
\hline \multicolumn{2}{|c|}{ Limits of application } & \multicolumn{2}{c|}{ Physical data } \\
\hline Max. velocity & $380 \mathrm{~km} / \mathrm{h}$ & $\begin{array}{l}\text { Nominal friction } \\
\text { coefficient }\end{array}$ & $0.35-0.40$ \\
\hline Max. pressure & $120 \mathrm{~N} / \mathrm{cm}^{2}$ & Thermal Conductivity & $39 \mathrm{~W} / \mathrm{mK}$ \\
\hline Max. permanent temperature & $700^{\circ} \mathrm{C}$ & Specific thermal capacity & $0.46 \mathrm{~J} / \mathrm{gK}$ \\
\hline $\begin{array}{l}\text { Max. instantaneous } \\
\text { temperature }\end{array}$ & $900^{\circ} \mathrm{C}$ & Hardness Brinell & 22 \\
\hline
\end{tabular}

During a single run six brake procedures with the same clamping force and effective mass are carried out for varying initial velocities. The chosen dataset consists of three runs with different clamping forces, yielding 18 load cases, as shown in Table 3. A scaled representation of train velocity $v$, clamping force $F_{C}$, mean disc temperature of all 6 measurement positions $T_{D}$ and acting friction coefficient $\mu$ of each run is depicted in Figure 4, Figure 5 and Figure 6. In order to guarantee similar initial conditions, the initial disc temperature needs to fall below a certain value before the next brake process is performed during a single run. For presentation purposes the time axis in the graphs is reduced to the brake processes. The cooling process of the brake disc is therefore not visible in these figures. 
In all three runs the friction coefficient strongly increases during brake applications with initial velocities below $100 \mathrm{~km} / \mathrm{h}$, resulting in a change between initial and final values of more than $30 \%$. At higher velocities a weakening of growth is observed during the first half of the brake process. This impact increases with larger clamping forces and initial velocities. Simultaneously, the mean disc temperature increases with larger clamping forces and initial velocities, due to a higher amount of energy which is transformed during braking. In case of $200 \mathrm{~km} / \mathrm{h}$ and $55 \mathrm{kN}$ the maximum mean temperature is reached and a reduction of the friction coefficient during the first half of the brake process is identified. The evolution of the estimated instantaneous friction coefficient on the test rig shows qualitative similarities to the measured train deceleration curves in Figure 1. This observation supports the hypothesis in which the friction forces are assumed to be responsible for the characteristic behavior of the measured deceleration curves of the train.

Table 3: Runs including measurement data of brake applications for different load cases

\begin{tabular}{|c|c|c|c|}
\hline Run & $F_{C}[\mathrm{kN}]$ & $m_{\text {eff }}[\mathrm{kg}]$ & $v_{0}[\mathrm{~km} / \mathrm{h}]$ \\
\hline 1 & 55 & 10000 & $50,80,120,140,160,200$ \\
\hline 2 & 36 & 10000 & $50,80,120,140,160,200$ \\
\hline 3 & 18 & 10000 & $50,80,120,140,160,200$ \\
\hline
\end{tabular}




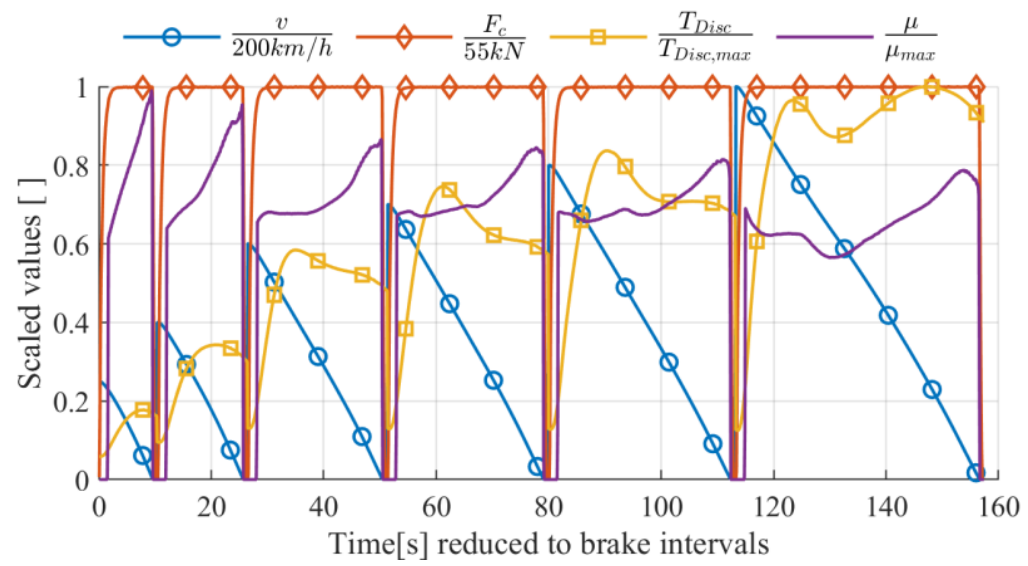

Figure 4: Scaled representation of measured data Run 1: $F_{C}=55 \mathrm{kN}, v_{0}=50,80,120,140,160,200 \mathrm{~km} / \mathrm{h}$

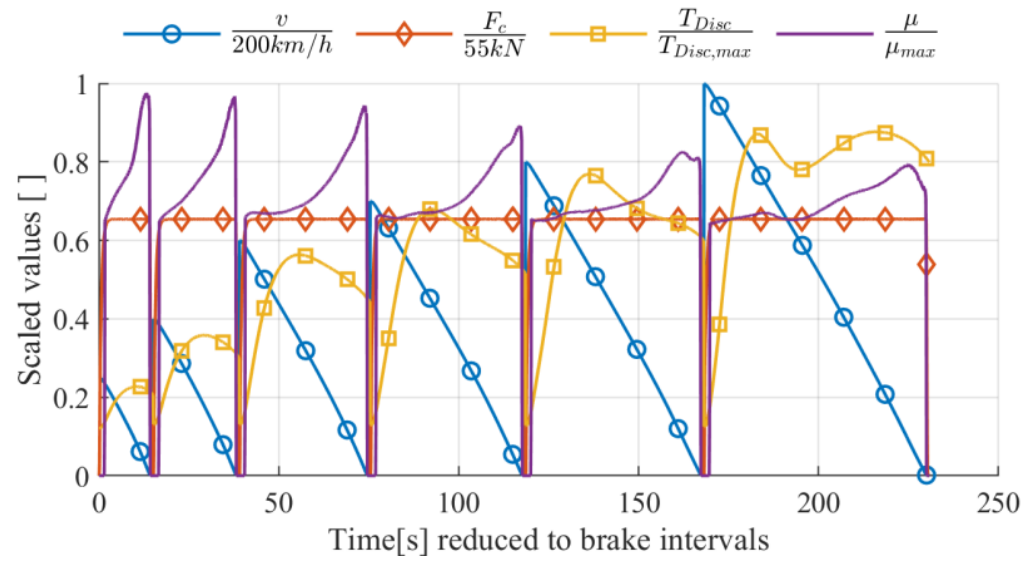

Figure 5: Scaled representation of measured data Run 2: $F_{C}=36 \mathrm{kN}, v_{0}=50,80,120,140,160,200 \mathrm{~km} / \mathrm{h}$

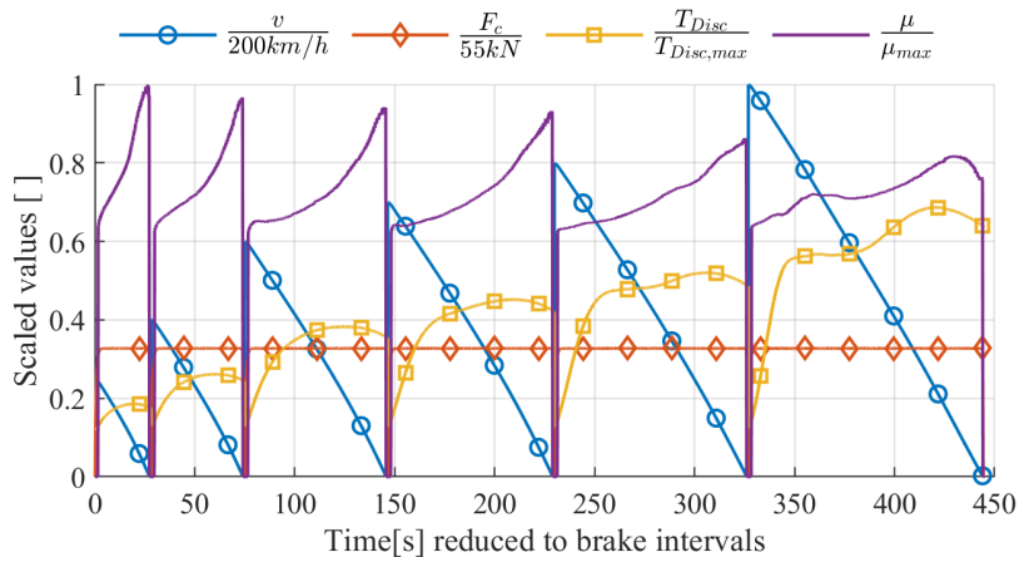

Figure 6: Scaled representation of measured data Run 3: $F_{C}=18 \mathrm{kN}, v_{0}=50,80,120,140,160,200 \mathrm{~km} / \mathrm{h}$ 


\section{Model Identification}

\subsection{Identification of factors influencing the instantaneous friction coefficient}

Applying the theory behind the chosen model approach, the observed behavior of the friction coefficient is a result of processes affecting growth, destruction and adhesion properties of the contact patches described by a differential equation. In scope of this work, it was not possible to analyze the surface topography of the pads and measure actual patch sizes. Therefore, the identification of factors primarily influencing the processes in the frictional boundary layer of the examined brake pad is based on a rather practical approach. Different factors, proposed in [6], [7] and [18], are systematically integrated in a differential equation according to the suggested formula in Table 1. By applying numerical integration, the instantaneous friction coefficient is simulated and qualitatively compared to the measured curve. Based on this procedure it is concluded that friction power $\mu \cdot F_{C} \cdot v_{F r}$, disc temperature $T_{D}$, clamping force $F_{C}$ and the friction coefficient $\mu$ itself determine the characteristic behavior of the investigated brake pad. Besides the identification of these factors, it is assumed that their influence on the change of the friction coefficient is generally nonlinear. Therefore, the following nonlinear differential equation is suggested:

$$
\dot{\mu}=-a \cdot\left(\mu \cdot F_{C} \cdot v_{F r}\right)^{x}-b \cdot \mu^{y}-c \cdot\left(\mu \cdot T_{D}\right)^{z}+d \cdot F_{C}^{r}
$$

The contribution of each factor is weighted with the coefficients $a, b, c$ and $d$ and the exponents $x, y, z$ and $r$, resulting in 8 parameters for the proposed model.

\subsection{Model interpretation}

Since the gradient of the friction coefficient is nearly constant at low initial velocities, a constant term is assumed to be responsible for the growth of the friction coefficient. In equation (8) this term is $d \cdot{F_{C}}^{r}$, which dependents on the mostly constant clamping force. The author assumes that increasing clamping forces lead to higher temperatures in the frictional boundary layer, which stimulates the growth of patches. A direct correlation between $\dot{\mu}$ and the actual patch temperature, as proposed in [6] and [18], is not considered in equation (8). The measurements provided for this work only contain data of the disc temperature close to the surface, which does not represent the temperature in the frictional boundary layer. 
An estimation of the patch temperature $\dot{T}_{P}$ during the brake process based on two parameters $\delta$ and $\varepsilon$ is given in [18]:

$$
\dot{T}_{P}=-\delta \cdot\left(T_{P}-T_{D}-\varepsilon \cdot F_{C} \cdot v_{F r}\right)
$$

However, parameters and results of equation (9) cannot be identified and verified with the available data. Furthermore, the correlation between $\dot{\mu}$ and an estimated patch temperature based on equation (9) yielded dynamics of the friction coefficient which do not match with the measured behavior. It is concluded that the indirect consideration of a stationary patch temperature via clamping forces is sufficient to depict the growth of $\mu$.

Friction power $\mu \cdot F_{C} \cdot v_{F r}$, friction coefficient $\mu$ itself and disc temperature $T_{D}$ are the identified variables which contribute to the reduction of the friction coefficient. As described in [6] and [7] mechanical loads drive the destruction of contact patches in the boundary layer. These loads are correlated to the transmitted friction power. Additionally, it is assumed that the size of the patches influences their stability [6]. Since the size of the total patch area is correlated to the friction coefficient, an enlargement of the coefficient yields reinforced patch destruction. The term including the disc temperature represents the so-called fading effect, as proposed in [18]. It is presumed that a change of the material properties due to high disc temperatures leads to a reduction of the friction coefficient [19]. In this case, a lag behavior of this term is identified, which can be recognized by the coupling of the disc temperature to $\mu$.

\subsection{Identification of model parameters}

For the identification of model parameters, a numerical optimization is performed, which aims to minimize the area arising from the deviation of measured and simulated friction coefficient by tuning the values of the parameters. In order to work with easy to handle values, the variables $T_{D}, \mu \cdot F_{C} \cdot v$ and $F_{C}$ in equation (8) are scaled to reference values. For the optimization task the Optimization Library for Dymola, described in [20] is used.

A multi-case optimization is performed in which the parameters of the nonlinear model are fitted for all three runs simultaneously. The objective function $f_{O b 1}$ is the sum of the squared integrated deviation between measured and simulated friction coefficient of all 18 brake processes $(i=1-18)$ over time. Each 
deviation integral is normalized to the length of the brake interval $T_{i}$ in order to guarantee the same weight for each brake process regarding the summed criterion:

$$
f_{o b 1}=\sum_{i=1}^{18}\left(\frac{1}{T_{i}} \int_{t_{0 i}}^{t_{\text {stop }, i}}\left|\mu_{m, i}(t)-\mu_{s, i}(t)\right| d t\right)^{2}
$$

The identified parameters are shown in Table 4. The start value is set to be the same for each simulation $\mu_{0}=$ const. This value represents the mean value of the measured friction coefficient at the beginning of each brake application, when the maximum pad force is reached. The scaled comparisons of the measured friction coefficient $\mu_{\text {measured }}$ and simulated friction coefficient $\mu_{\text {model } 1}$ for run 1, 2 and 3 are shown in Figure 7, Figure 8 and Figure 9 (blue continuous and red dash/dotted lines). Except for the first and third brake process of run 1 and the last brake processes of run 2 and 3, the evolution of the friction coefficient of the simulated model is well represented. This means that the identified model is able to reproduce 14 of 18 different load cases using a single set of parameters. Larger deviations occur in brake processes with an initial velocity of $200 \mathrm{~km} / \mathrm{h}$ and clamping forces smaller $40 \mathrm{kN}$. It is assumed that the predominant processes in the frictional boundary layer are not considered properly by the model in case of these brake applications.

Table 4: Identified model parameters for all runs based on criterion $\boldsymbol{f}_{o b 1}$

\begin{tabular}{|c|c|c|c|c|}
\hline Parameters & $\mathrm{a}$ & $\mathrm{b}$ & $\mathrm{c}$ & $\mathrm{d}$ \\
\cline { 2 - 5 } (Coefficients) & 0.01 & 0.02 & $8 \mathrm{e}-5$ & 0.04 \\
\hline Parameters & $\mathrm{x}$ & $\mathrm{y}$ & $\mathrm{z}$ & $\mathrm{r}$ \\
\cline { 2 - 5 } (Exponents) & 0.6 & 0.4 & 0.9 & 0.3 \\
\hline
\end{tabular}




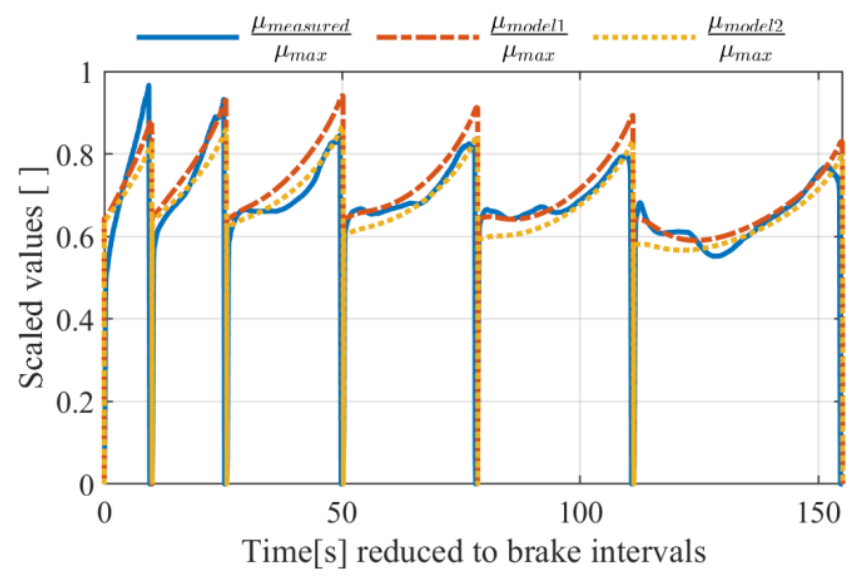

Figure 7: Scaled representation of measured and simulated $\mu$

Run 1: $F_{C}=55 \mathrm{kN}, v_{0}=50,80,120,140,160,200 \mathrm{~km} / \mathrm{h}$

( $\mu_{\text {model } 1}$ based on criterion $\boldsymbol{f}_{o b 1}$ and $\mu_{\text {model } 2}$ based on criterion $\boldsymbol{f}_{o b 2}$ )

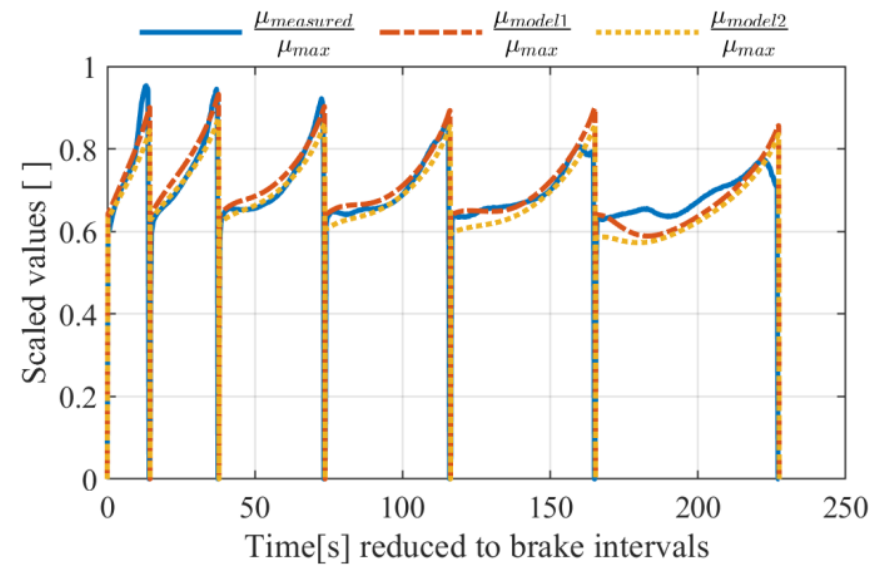

Figure 8: Scaled representation of measured and simulated $\mu$

Run 2: $F_{C}=36 \mathrm{kN}, v_{0}=50,80,120,140,160,200 \mathrm{~km} / \mathrm{h}$

( $\mu_{\text {model } 1}$ based on criterion $\boldsymbol{f}_{o b 1}$ and $\mu_{\text {model } 2}$ based on criterion $\boldsymbol{f}_{o b 2}$ )

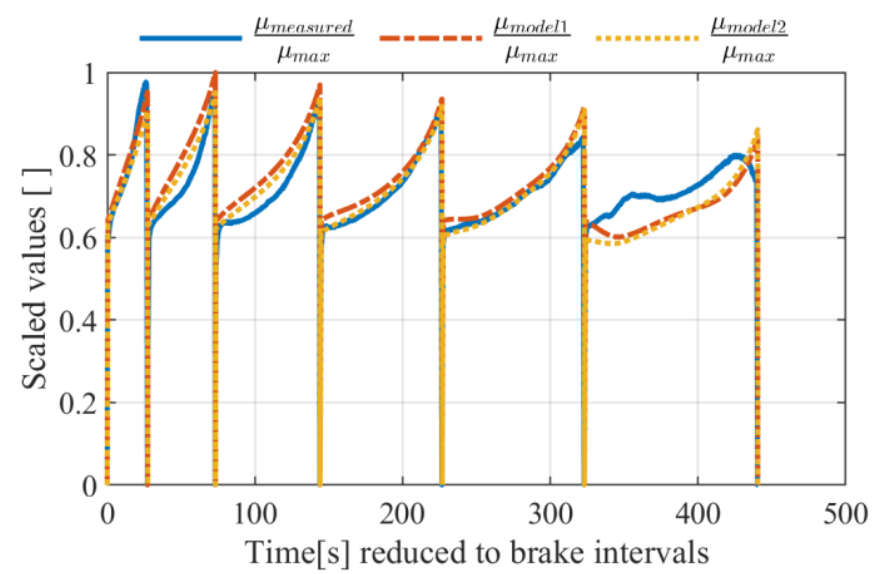

Figure 9: Scaled representation of measured and simulated $\mu$

Run 3: $F_{C}=18 \mathrm{kN}, v_{0}=50,80,120,140,160,200 \mathrm{~km} / \mathrm{h}$

( $\mu_{\text {model } 1}$ based on criterion $\boldsymbol{f}_{o b 1}$ and $\mu_{\text {model } 2}$ based on criterion $\boldsymbol{f}_{o b 2}$ ) 


\section{Model Verification}

Finally, the identified model is verified regarding its suitability for the estimation of the brake distance. The curves $\mu(t)$ from the multi-case optimization, which took all runs into account, are used to calculate the brake distance $s$ by applying numerical integration for the time interval $\left[t_{0}, t_{\text {stop }}\right]$ :

$$
s=-\iint_{t_{0}}^{t_{\text {stop }}}\left(\frac{\mu(t) \cdot r_{F r} \cdot F_{C}(t)}{m_{\text {eff }}}\right) d t d t+v_{0} \cdot t
$$

Additionally, the brake distance is calculated based on (11) using a constant value for the friction coefficient, $\mu(t)=\mu_{\text {mean }}$. This value corresponds to the mean friction value of all 18 brake applications with respect to the distance $s_{0.95}$ measured from the point of time at which $95 \%$ of the maximum normal force is acting [21]:

$$
\mu_{\text {mean }}=\frac{1}{18} \sum_{i=1}^{18}\left(\frac{1}{s_{0.95, i}} \int_{0}^{s_{0.95, i}} \mu_{i}(t) d s\right)
$$

In Figure 10 the relative deviation between calculated and measured brake distance using both approaches for $\mu(t)$ are compared (blue and red bars). As one can see in the legend, the mean deviation over all runs is nearly the same for both approaches, $\Delta s \approx 2.5 \%$. For the brake applications $1,2,6,7,8,9,13$, and 17 the use of the model with variable $\mu(t)=\mu_{\text {model } 1}$ performs better than $\mu_{\text {mean }}$. The maximum deviation of both models is about $8 \%$. The deviation of the friction coefficient at high velocities has a larger impact on the difference of the brake distance than errors at low velocities. This means that simulated curves that coincide better with the measured friction value at the beginning of the brake interval lead to a smaller deviation. In comparison to equation (12), the criterion $f_{O b 1}$ of the performed multi-case optimization does not weight the deviation of the friction coefficient with respect to distance. However, the model yields a comparable accuracy and furthermore allows for an appropriate estimation of the instantaneous friction forces during the entire brake process. The latter is not possible with a constant mean value for $\mu(t)$.

It becomes apparent that the choice of the optimization criterion influences the accuracy of the identified model regarding the brake distance. Furthermore, the choice of an appropriate initial value $\mu_{0}$ is crucial for the estimation of the brake distance, since this value is present at the beginning of the brake process. By 
weighting the deviation of the friction coefficient with the instantaneous velocity $v(t)$ it is possible to assign the criterion of the objective function regarding the brake distance:

$$
f_{O b 2}=\sum_{i=1}^{18}\left(\frac{1}{T_{i}} \int_{t_{0 i}}^{t_{\text {stop } i}}\left|\mu_{m, i}(t)-\mu_{s, i}(t)\right| \cdot v(t) \cdot d t\right)^{2}
$$

Using this objective function and introducing $\mu_{0}$ as parameter that is additionally tuned during optimization, a mean deviation of the brake distance of $2.1 \%$ with a maximum deviation lower than $6 \%$ is reached (Figure 10 , yellow bars of $\mu_{\text {model } 2}$ ). A smaller maximum deviation allows for a reduction of the safety margin when using the model for calculation of brake distances. However, this model might not provide appropriate information for the instantaneous friction forces, as shown in Figure 7, Figure 8 and Figure $9\left(\mu_{\text {model } 2}\right.$, yellow dotted line). Thus, the choice of the optimization criterion depends on the application of the model and its simulation results.

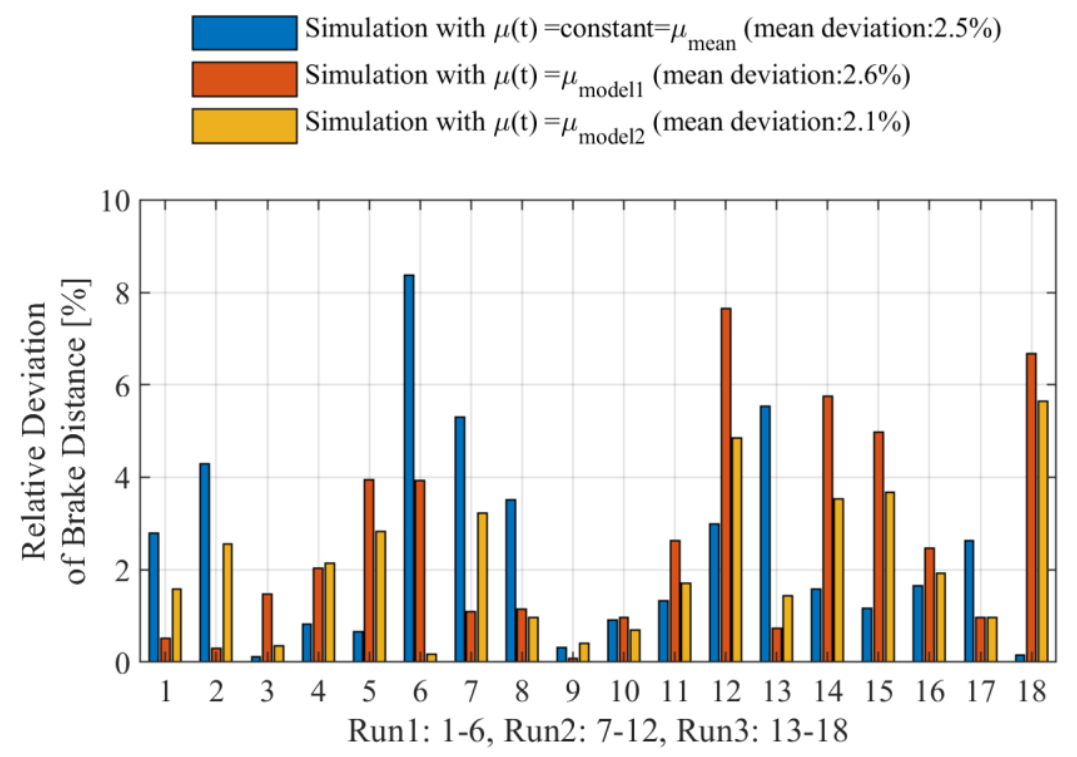

Figure 10: Deviation of measured and simulated brake distance based on use of constant mean friction coefficient $\left(\mu_{\text {mean }}\right)$ and estimated instantaneous friction coefficient $\left(\mu_{\text {model1 }}, \mu_{\text {model } 2}\right)$ 


\section{Conclusion and Outlook}

In this work a dynamic model for the friction coefficient acting in railway disc brakes is developed. In comparison to common approaches applied for railway vehicles the presented method is based on processes taking place in the frictional boundary layer. This yields a differential equation correlating the instantaneous friction coefficient to external loads. Measurements recorded on a full-scale dynamometer test rig are used for the model identification. These measurements support the hypothesis that the change in frictional properties is considered to be the cause for the varying deceleration of the train during emergency brake applications shown in Figure 1. For the examined sintered brake pad, specified in Table 2, it is concluded that the clamping force is primarily responsible for the rise of the instantaneous friction coefficient, whereas friction power, disc temperature and the coefficient itself are the predominant variables affecting the decrease of the friction coefficient. Furthermore, it is found that the relationship between the variables and the change of the friction coefficient is in general nonlinear.

The model parameters are identified by applying numerical optimization. The resulting simulated friction coefficient shows good accordance to the measurements for most cases using a single set of parameters. Larger deviations occur for load cases with an initial velocity of $200 \mathrm{~km} / \mathrm{h}$. The application of the simulated curves for the estimation of the brake distances leads to a mean deviation of $2.6 \%$ compared to the measurements on the test rig. This accuracy is comparable to the use of a constant mean value for the friction coefficient with respect to distance $(2.5 \%)$. It is possible to improve the accuracy of the model regarding the brake distance by weighting the optimization criterion with the instantaneous velocity. This leads to a mean deviation of $2.1 \%$ and a maximum deviation less than $6 \%$. Based on the results it is concluded that the chosen dynamic model approach is suitable for the simulation of instantaneous friction forces in railway disc brakes.

The results of this work motivate further investigations. Initially, the findings need to be verified by a more detailed analysis of the pad surface and its frictional properties during braking. An investigation of the pad topography before and after brake procedures on the test rig, as done in [11], seems to be a promising method to understand the processes taking place in the frictional boundary layer of railway disc brakes. 
Furthermore, an exchange with experts dealing with frictional materials of disc brakes is necessary to assess the results.

It is important to mention that the processes acting in the frictional boundary layer strongly depend on the brake components, especially the brake pad and its material. In this work a sintered material has been investigated. The processes acting in other common pad materials applied in railway applications, such as organic materials, might be different and the suggested model might not be applicable. Thus, the identification needs to be extended to provide models for different pad materials as well as disc types and environmental conditions. This includes the development of a valid model for the estimation of the disc temperature. Applying the method suggested in this work for common types of brake pads and discs might lead to a broader and deeper understanding of the friction processes in railway disc brakes. This knowledge is indispensable for the system optimization and further development of friction brake systems, such as electro-mechanic and controlled friction brakes. Furthermore, enhanced friction models can be applied in online control strategies which are based on the real time estimation of frictional properties, as presented in [22].

Finally, the model of the friction coefficient needs to be linked to models for the disc temperature, brake actuation, wheel-rail adhesion as well as longitudinal dynamics to estimate the deceleration of entire railway vehicles during braking. In order to use these models for virtual homologation tests, the simulation results need to be verified with measured data from field tests, as shown in Figure 1 and Figure 2 . The verified models can be applied to analyze large numbers of emergency brake applications as well as route profiles, regarding brake distance, temperature evolution in discs and resulting wear. Furthermore, the models can be used to develop and assess control algorithms for the actuation of frictional and electrodynamic brake systems during various operational brake scenarios. This includes prospective operational scenarios, such as moving in relative brake distance, that aim to increase traffic volumes on the existing infrastructure. 


\section{Acknowledgement}

This work is partly sponsored by Knorr-Bremse SfS GmbH. I would like to thank all colleagues from KnorrBremse for their strong support during the common project. This includes the exchange of expertize as well as the provision of measurement data, which is indispensable for this work.

\section{Declaration of conflicting interests}

The author(s) declared no potential conflicts of interest with respect to the research, authorship, and/or publication of this article.

\section{References}

1. DIN 14531-1. Railway applications - Methods for calculation of stopping and slowing distances and immobilisation braking - Part 1: General algorithms utilizing mean value calculation for train sets or single vehicles. 2009.

2. DIN 14531-2. Railway applications - Methods for calculation of stopping and slowing distances and immobilisation braking - Part 2: Step by step calculations for train sets or single vehicles. 2016.

3. Cantone L and Ottati A. Modelling of Friction Coefficient for Shoes Type LL By Means of Polynomial Fitting. The Open Transport Journal. 2018: 12, pp. 114-127.

4. Wende D. Fahrdynamik des Schienenverkehrs. 1st ed. Heidelberg: Vieweg+Teubner Verlag, 2003.

5. Lee NJ and Kang CG. The Effect of a Variable Disc Pad Friction Coefficient for the Mechanical Brake System of a Railway Vehicle. PLOS ONE. 2015; 10: pp. 1-18

6. Ostermeyer GP. On the dynamics of the friction coefficient. Wear 2003; 254: pp.852-858.

7. Ostermeyer GP. Eigenschaften der Reibpaarungen im Bremsprozess. In: Breuer B and Bill KH Bremsenhandbuch. 5th ed. Wiesbaden: Springer Vieweg, 2017, pp. 593-609.

8. Popov VL. Kontaktmechanik und Reibung. 2nd ed. Heidelberg: Springer-Verlag, 2010.

9. Berger EJ. Friction modeling for dynamic system simulation. Applied Mechanics Reviews. 2002; 55: pp. 535-577.

10. Olsson H, Aström KJ, Canudas de Wit C, et al. Friction Models and Friction Compensation. European Jounal of Control. 1997, pp. 176-195.

11. Ostermeyer GP and Wikening L. Experimental Investigation of the Topography Dynamics in Brake Pads. SAE International Journal of Passenger Cars-Mechanical Systems. 2013; 6: pp: 1398-1407

12. Ricciardi V, Augsburg K, Gramstat S, et al. Survey on Modelling and Techniques for Friction Estimation in Automotive Brakes. Applied Science. 2017; 7: p. 873

13. Rhee SK. Friction Coefficient of Automotive Friction Materials - Its Sensitivity to Load, Speed, and Temperature. SAE Technical Paper. 1974;

14. Loh WY, Hecht Basch R, Li D, et al. Dynamic Modeling of Brake Friction Coefficients. SAE Technical Paper. 2000; 
15. Ćirović V, Aleksendrić D. Development of Neural Network Model of Disc Brake Operation. FME Transactions. 2010; 38: pp. 29-38.

16. KNORR-BREMSE Systeme für Schienenfahrzeuge GmbH. Basics of Brake Technology. 2nd ed. München: KNORR-BREMSE GmbH, 2003.

17. UIC-Kodex. 541-3 Brakes - Disc brakes and their application - General conditions for the certification of brake pads. 2017.

18. Ostermeyer GP and Bode P. On Dynamic Friction Phenomena in Brake Systems. In: Fischer A and Bobzin K Friction, Wear and Wear Protection. Wiley-VCH Verlag GmbH \& Co. KGaA, 2009; pp. 301306.

19. Remfrey J, Gruber S and Sendler J. Aufbau und Komponenten von Pkw-Bremsanlagen. In: Breuer B and Bill KH Bremsenhandbuch. 5th ed. Wiesbaden: Springer Vieweg, 2017, pp. 123-181.

20. Pfeiffer A. Optimization Library for Interactive Multi-Criteria Optimization Tasks. In: Proceedings of the 9th International Modelica Conference. München, Germany, 3-5 September, 2012, pp. 669-679

21. UIC-Kodex. UIC 548 Brakes - Requirements of friction test benches for the international certification of brake pads and brake. 2016.

22. Heckmann A, Schwarz C, Keck A and Bünte T. Nonlinear Observer Design for Guidance and Traction of Railway Vehicles. In: Proceedings of the 26th IAVSD International Symposium on Dynamics of Vehicles on Roads and Tracks, Gothenburg, Sweden, 12-16 August, 2019, pp. 639-648 